\section{BMJ Open Respiratory Research}

\title{
Democratic and ethical problem of lung cancer screening: exclusion of true high- risk populations. Can it be fixed? Yes
}

\author{
Oluf Dimitri Røe (1) 1,2
}

\begin{abstract}
To cite: Røe OD. Democratic and ethical problem of lung cancer screening: exclusion of true high-risk populations. Can it be fixed? Yes. BMJ Open Resp Res 2020;7:e000811. doi:10.1136/ bmjresp-2020-000811
\end{abstract}

Received 23 October 2020 Revised 29 November 2020 Accepted 30 November 2020

D Check for updates

(C) Author(s) (or their employer(s)) 2020. Re-use permitted under CC BY-NC. No commercial re-use. See rights and permissions. Published by BMJ.

${ }^{1}$ Department of Clinical and Molecular Medicine, Norwegian University of Science and Technology, Trondheim, Norway ${ }^{2}$ Cancer Clinic, Levanger Hospital, Nord-Trondelag Health Trust, Levanger, Norway

Correspondence to Dr Oluf Dimitri Røe; olufdroe@yahoo.no

\section{ABSTRACT}

Screening a population for a potentially deadly disease, the ultimate goal must be to prevent morbidity and mortality from this disease for the whole population. Unlike breast cancer or cervical cancer screening, where all women are screened after a certain age, CT screening for lung cancer has been based on selection of putative high-risk individuals based on age and smoking cut-off values. The type of selection used leaves too many high-risk individuals behind. The solution is to use only validated risk prediction models for selection.

Early detection of lung cancer is currently the most effective way to reduce the total mortality of lung cancer. Patients with lung cancer diagnosed at stage I have a 5-year survival of more than $80 \%$ while the total overall survival is $15 \%-20 \%$. $^{1}$ Lung cancer screening by CT is currently the only method to diagnose lung cancer in an early stage, as blood biomarkers are still not widely validated or used. ${ }^{2}$

CT screening has been shown to reduce lung cancer mortality by $20 \%$ in all and $24 \%$ in men in large prospective studies as the NLST (USA) published in 2011 and NELSON (Belgium, Netherlands) in $2020,{ }^{3}{ }^{4}$ respectively. CT screening has some important side effects that are widely discussed, including false positive or biologically inert cancer nodules requiring unnecessary interventions, anxiety, the cancer risk with repeated CT scans, cost-effectiveness and the economic burden of the health system. Those questions have hindered a wide acceptance of CT screening in Europe. But what is the most serious problem of lung cancer screening? Probably that it excludes most of the population at true high risk and includes too many with low risk.

When we talk about high-risk populations, we tend to refer to heavy smokers of older age. The fact is that a large population with true high risk will never be included in screening programmes because they do not fit the commonly used high-risk inclusion criteria. The criteria for inclusion in the NLST were age $55-74$ years, $>30$ pack-years and $<15$ years quit time. These criteria resulted in an estimated inclusion of only $26.7 \%$ of those who develop lung cancer in the general population in the USA, missing out $73 \%$ of future patients with lung cancer. ${ }^{5}$ A gain of $20 \%$ survival in the $26.7 \%$ of those developing lung cancer may be seen as a success, but in the total population of patients with lung cancer this is less impressive. Based on these figures, for example, out of 1000 patients developing lung cancer, 267 would be eligible for the NLST screening programme and of those, 54 (20\%) with benefit, a net survival benefit of $5.5 \%$. But the benefit is even less if you do not include all in the target group. In the USA, where CT screening for lung cancer has been approved and reimbursed for several years, it has been underused, due to social disparities and other factors. ${ }^{6}$

Norway is a country where people do not smoke too much. Still, one person dies from lung cancer every 4 hours. Norway has no screening programme yet. The HUNT2, a large Norwegian population study that included more than 65000 individuals age above 20 years during 1995-1996 that were followed for more than 15 years, had smoked median pack-years of 10.3 atinclusion. ${ }^{7}$ Among those, $64 \%$ of ever-smokers developing lung cancer would be excluded from screening by the NLST criteria, simply because they had smoked less than 30 years at baseline. ${ }^{7}$ The NLST criteria also omits younger and older people at risk. In another large Norwegian cohort, CONOR, with more than 45000 eversmokers, followed for a median of 11.6 years, among ever-smokers developing lung cancer, $21.35 \%$ were younger than $55 \%$ and $18.41 \%$ were above 74 at baseline, indicating that the NLST age cut-offs by itself exclude almost $40 \%$ of the eligible population. ${ }^{7}$ Clearly, the NLST criteria are insufficient for selecting the true high-risk population, in the USA and in Norway (figure 1). 
Similarly, the NELSON study included persons of age $50-75$ years, $>15$ cigarettes a day $>25$ years $(>18.75$ packyears), or $>10$ cigarettes a day $>30$ years ( $>15$ pack-years), 10 years or less quit time. How large portion at risk did not fit the criteria? Unknown. However, they cannot compete with the risk models. When we compared a 'Reduced' HUNT model with the NELSON criteria, in a heavy smoker Danish population, one would need to screen 26\% more people with the NELSON criteria to detect the same number of lung cancers. ${ }^{8}$

So, in short, the gain from screening in its current form is minuscule in a real-life population due the exclusion of at-risk individuals. ${ }^{8}$

This problem has been approached by the US Preventive Services Task Force by widening the inclusion criteria to age $50-80$ years, and lower the smoking burden to 20 pack-years.

Still this will exclude a large high-risk population. In the CONOR population, among those developing lung cancer, 37\% had smoked less than 20 pack-years at baseline. ${ }^{7}$ Moreover, a simple change of the age and smoking burden will just add a large number of true low-risk individuals that will undergo unnecessary screening.

But why are all those individuals at risk excluded by these seemingly reasonable age and smoking cut-offs? Among patients with lung cancer in the CONOR population, $95.5 \%$ were current or former smokers, but among all current or former smokers, only $0.6 \%$ developed lung cancer in a follow-up of 6 years. This means that since most smokers never get lung cancer, the risk for an individual depends on a combination of biological factors based on genes, lifestyle and environmental exposures. ${ }^{9}$ To exemplify, while one person may smoke for 10 years and develop a lung cancer at the age of 45 years, another can smoke 20 a day for 60 years and never get cancer. Risk is based on a complex interplay of these factors.

The good news is that personal risk for lung cancer can be calculated. The current validated risk prediction models have repeatedly shown that they are superior in selecting people for lung cancer screening than by using the simple cut-offs. ${ }^{78}{ }^{10-13}$ The use of risk prediction models, by reducing the number of screened individuals, is also cost-effective. ${ }^{8}$ Not only that. Because they produce a ranking of individuals according to risk, one must set a risk threshold above which screening should take place. The threshold decides how many will be screened and the portion of lung cancers that will be detected. This threshold can be decided by health authorities in each country considering all public health and economic factors. Screening policy will therefore be more transparent, objective and tailored to the local health system. Cost-effectiveness of a screening programme, in my view, depends on how expensive the CT screening is and how many unnecessary interventions are needed versus the

\section{Number of lung cancers in $\mathbf{4 5} \mathbf{3 8 7}$ ever-smokers in six years CONOR, NORWAY}

300

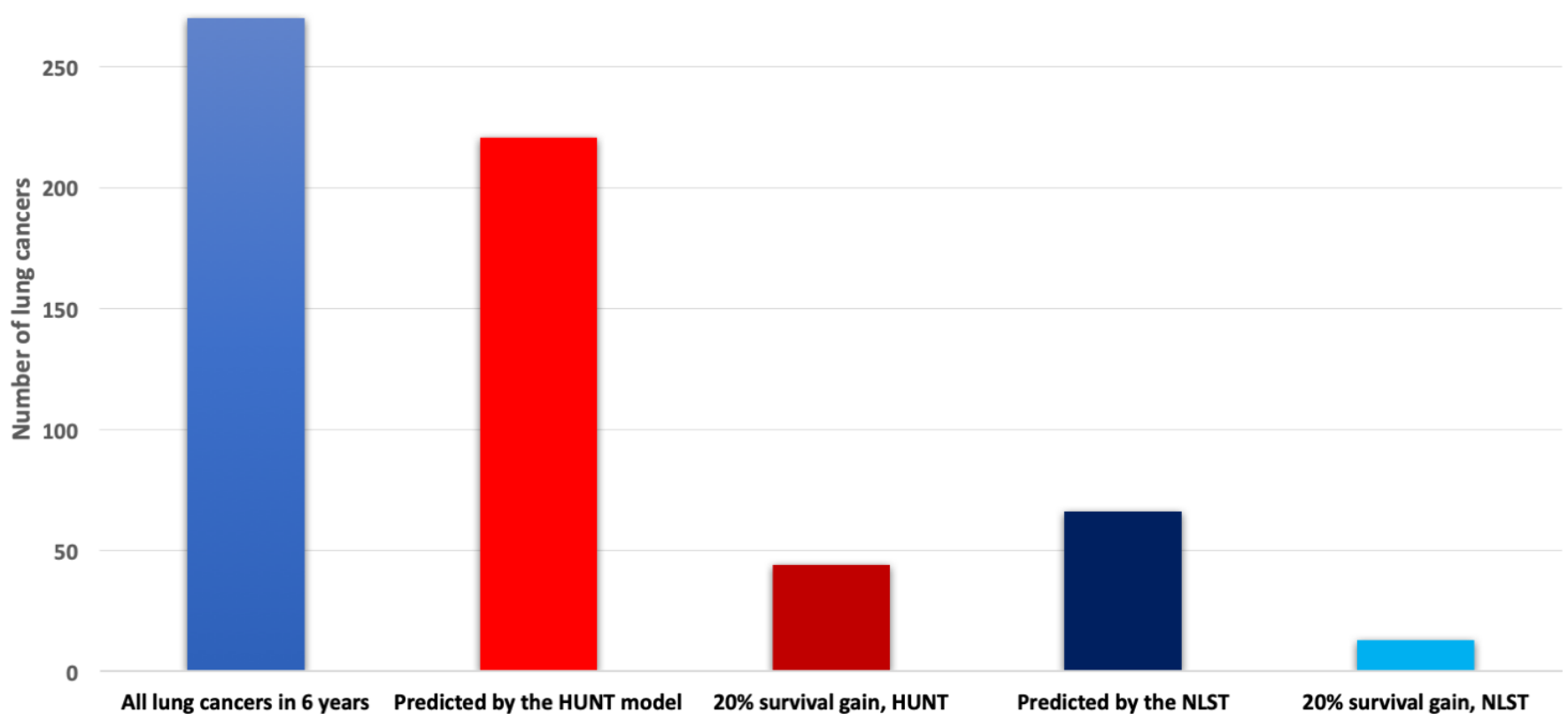

Figure 1 Performance of the HUNT Lung Cancer Model ${ }^{7}$ versus NLST criteria for lung cancer diagnosis within 6 years in the validation cohort CONOR of ever-smokers using as threshold the $16 \%$ quantile of risk of events in HUNT corresponding to a lung cancer risk at least $0.64 \%$ in 6 years. Only $22 \%$ of ever-smokers would need screening to identify $81 \%-85 \%$ of all lung cancers within 6 years. With the NLST criteria $24 \%$ would be identified. The $20 \%$ gain in survival by the HUNT would be $3.4-$ fold that of NLST. Data from ${ }^{7}$ with permission. 
gain for the person and the society by increased life expectancy and the avoidance of hurtful and costly lifeprolonging therapy. The effectiveness will also change based on this, so the risk threshold must be set to balance all these factors, for each country separately. However, as we found, using a risk model not only do we find significantly more cancers than the NLST and NELSON criteria, but when using the same sensitivity as these criteria in a population of smokers, we need to screen 20\%-30\% less individuals. So, it is actually a win-win situation. ${ }^{8}$

A clinical risk prediction model is imperative for the best selection of individuals for screening, as has been noted in a European Union position statement published in the Lancet Oncology in $2017^{14}$ and reiterated in $2020 .{ }^{15}$ There is therefore positive attitude by the screening community towards risk prediction models. However, so far, they have not been used in prospective screening studies.

One concern has been that risk models may choose a predominantly older population. One study on a simulated smokers population found that using a risk model did not increase the life-years (all causes of death included) and they concluded that this was due to the risk model selecting older people at risk. ${ }^{16}$ However, this solid study leans heavily on a smoke imputation model based on a population in the USA starting from 1890 . Smoking history data are difficult to obtain correctly, and prospective studies are the most reliable. When using our HUNT Lung Cancer Model in the prospective $>45000$ ever-smokers CONOR population, it detected $>80 \%$ of lung cancers in 6 years. We found that the median age of the selected individuals at risk was 61 years, and among those developing cancer 67 years. With the NELSON criteria in the same population, the median age was 60 years and 61 years, respectively, but they only detected $35 \%$ of lung cancers. Is it ethical and democratic to exclude a so large population with a potentially deadly disease from a screening programme?

Moreover, when you screen for other cancers, like breast or cervical cancer in women, you do not look for competing causes of death as the benchmark, these causes may vary greatly between populations. Importantly, one should use clinical insight and not include people in a screening programme that cannot receive curative treatment. However, this decision can be taken face to face, just as an anaesthesiologist decides if you are fit for surgery, not by general, a priori decisions.

The next question is, which model to use. Among the more than 20 models that have been developed, only few have been validated, and among the researchers there are disputes on which is the best model. ${ }^{13}$ Currently the LPPi, PLCOm2012 and the HUNT Lung Cancer Model have been validated in large prospective populations. They all perform well, but there are important differences. Ideally, a model should be tested and validated in a complete population. Moreover, there should be a long follow-up time, a large age-span and people with all types of smoking behaviour. The model should be fit or tested in cohorts of light and heavy smokers of all ages. The variables should be easy to answer reliably by those screened. All these criteria have, to the best of my knowledge, only been fulfilled by the HUNT Lung Cancer Model. The HUNT Lung Cancer Model and risk calculator include seven simple variables: age, pack-years, smoking intensity (cigarettes per day), years since smoking cessation, body mass index, 'daily cough in periods of the year' and 'hours of indoors smoke exposure'. ${ }^{7}$ It also comes with an online calculator that is open for health professionals and the public. The HUNT and most other models were tested mostly in Caucasian populations, so there is not enough knowledge on how these models work in predominantly African, Asian or other populations. For example, it has been shown that African Americans may have a higher risk than Caucasian Americans, and Asian Americans have lower. However, is this a genetic, social, mixed component, and who should be labelled African American or Asian American? The best way to reassure that the genetic component is accounted for is probably not by the hue of the skin colour, but a blood test on risk genes, which is currently under study and will, at least from a technical point of view, be feasible in practice. ${ }^{17}$

The main goal of lung cancer screening should ideally be to identify most lung cancers in a timely fashion and to cure these patients by surgery or stereotactic radiotherapy only. This has been partly achieved with the current CT screening programmes, in several populations and in many countries. It is non-democratic and non-ethical to exclude more than half of those individuals that are in a verified risk group and not use the current validated tools to lift the bar and achieve screening of all with true high risk. Moreover, the only way to minimise harm is by excluding those with true low risk by validated risk models and calculators, and it is cost-effective. ${ }^{8}$ So, let the best risk models take a front seat in the future European, American and worldwide lung cancer screening programmes. It is long due.

Acknowledgements Thanks to Maria Markaki, University of Crete, Greece, for kind assistance in the revised version of this manuscript.

Contributors ODR is the sole author of this work and the viewpoints represent his own.

Funding This study was funded by The Liaison Committee between the Central Norway Regional Health Authority (RHA) and the Norwegian University of Science and Technology.

Competing interests None declared.

Patient consent for publication Not required.

Provenance and peer review Not commissioned; externally peer reviewed.

Open access This is an open access article distributed in accordance with the Creative Commons Attribution Non Commercial (CC BY-NC 4.0) license, which permits others to distribute, remix, adapt, build upon this work noncommercially, and license their derivative works on different terms, provided the original work is properly cited, appropriate credit is given, any changes made indicated, and the use is non-commercial. See: http://creativecommons.org/ licenses/by-nc/4.0/.

ORCID iD

Oluf Dimitri Røe http://orcid.org/0000-0002-4870-5822 


\section{REFERENCES}

1 Chansky K, Detterbeck FC, Nicholson AG, et al. The IASLC Lung Cancer Staging Project: External Validation of the Revision of the TNM Stage Groupings in the Eighth Edition of the TNM Classification of Lung Cancer. J Thorac Oncol 2017:12:1109-21.

2 Chu GCW, Lazare K, Sullivan F. Serum and blood based biomarkers for lung cancer screening: a systematic review. BMC Cancer 2018;18:181.

3 Aberle DR, Adams AM, et al, National Lung Screening Trial Research Team. Reduced lung-cancer mortality with low-dose computed tomographic screening. N Engl J Med 2011;365:395-409.

4 de Koning HJ, van der Aalst CM, de Jong PA, et al. Reduced lungcancer mortality with volume CT screening in a randomized trial. $N$ Engl J Med 2020;382:503-13.

5 Pinsky PF, Berg CD. Applying the National lung screening trial eligibility criteria to the US population: what percent of the population and of incident lung cancers would be covered? J Med Screen 2012;19:154-6.

6 Watson KS, Siegel LD, Henderson VA, et al. The shared project: a novel approach to engaging African American men to address lung cancer disparities. Am J Mens Health 2020;14:1557988320958934.

7 Markaki M, Tsamardinos I, Langhammer A, et al. A validated clinical risk prediction model for lung cancer in smokers of all ages and exposure types: a HUNT study. EBioMedicine 2018;31:36-46.

8 Røe OD, Markaki M, Tsamardinos I, et al. 'Reduced' Hunt model outperforms NLST and Nelson study criteria in predicting lung cancer in the Danish screening trial. BMJ Open Respir Res 2019;6:e000512.

9 Shields PG, Harris CC. Cancer risk and low-penetrance susceptibility genes in gene-environment interactions. J Clin Oncol 2000;18:2309-15.

10 Katki HA, Kovalchik SA, Berg CD, et al. Development and validation of risk models to select Ever-Smokers for CT lung cancer screening. JAMA 2016;315:2300-11.

11 Tammemägi MC, Katki HA, Hocking WG, et al. Selection criteria for lung-cancer screening. N Engl J Med 2013;368:728-36.

12 Tammemägi MC. Application of risk prediction models to lung cancer screening: a review. J Thorac Imaging 2015;30:88-100.

13 Røe OD. Answer/comment to Prof. Tammemagi's article "Selecting lung cancer screenees using risk prediction models-where do we go from here". Transl Lung Cancer Res 2019;8:192-4.

14 Oudkerk M, Devaraj A, Vliegenthart R, et al. European position statement on lung cancer screening. Lancet Oncol 2017;18:e754-66.

15 Kauczor H-U, Baird A-M, Blum TG, et al. ESR/ERS statement paper on lung cancer screening. Eur Radiol 2020;30:3277-94.

16 Ten Haaf K, Bastani M, Cao P, et al. A comparative modeling analysis of Risk-Based lung cancer screening strategies. J Natl Cancer Inst 2020;112:466-79.

17 Røe OD, Nguyen OTD, Lakiotaki K, et al. Improvement of lung cancer risk prediction adding SNPs to the HUNT lung cancer model: a HUNT study. JCO 2019;37:e20696-e. 